\title{
Foreword: Sacral nerve stimulation now and in the future
}

\author{
Charles W. Butrick
}

Published online: 23 October 2010

(C) The International Urogynecological Association 2010

Normal bladder function depends on the integrity of the nervous system. Even minor alterations in this complex neurologic control can lead to many of the commonly seen bladder disorders. These often subtle dysregulations in neurologic function can result in life changing dysfunction of the pelvic floor, bladder and bowel.

This supplement was assembled as a resource for the clinician who cares for patients with pelvic floor dysfunction, urge incontinence, urinary frequency, urinary retention and bowel dysfunction. It includes a review of the history and the mode of action for neuromodulation, tips on patient selection as well as techniques and outcomes of sacral neuromodulation. Many of our patients who do not respond to more traditional therapies can benefit with this modality. Sacral neuromodulation is a well-established treatment and has been widely used since the original Food and Drug Administration (FDA) approval in 1997. It has evolved into a highly successful, minimally invasive therapeutic approach that should be offered to patients who have failed to respond to traditional therapies. Clinicians using sacral neuromodulation have started to push the envelope of indications and this supplement will also discuss some of these potential indications for which it might be used in the future. This supplement will hopefully stimulate the reader to consider sacral neuromodulation as a very viable option that should be offered to patients whose pelvic floor dysfunction has failed to respond to more traditional management.

Conflicts of interest None.

C. W. Butrick $(\bowtie)$

The Urogynecology Center,

12200 W. 106th Street, Suite 130,

Overland Park, KS 66215, USA

e-mail: cwbutrick@gmail.com 